\title{
Cyclical variation of blood pressure and heart rate in neonates
}

\author{
S Cunningham, S Deere, N McIntosh
}

\begin{abstract}
Using a computerised physiological monitoring system a cyclical variation in blood pressure (waves), with associated changes in heart rate and transcutaneous oxygen, was observed. Twenty five episodes were seen in 10 neonates, with a median gestation of 33 weeks (range 28-42 weeks). Eight neonates had an asphyxial injury.

Blood pressure waves had a mean (SD) amplitude of $11.6(5.6) \mathrm{mm} \mathrm{Hg}$ with a mean wavelength of $9.0(5 \cdot 2)$ minutes. Both amplitude and frequency were independent of gestation. In neonates of less than 34 weeks an inverse phase relation existed between heart rate and blood pressure waves (blood pressure rose as heart rate fell); in infants with a gestation greater than 34 weeks a direct phase relationship occurred (blood pressure and heart rate rose together).
\end{abstract}

It is postulated that hypertensive blood pressure waves may cause or exacerbate cerebral pathology in neonates with a pressure passive cerebral circulation.

(Arch Dis Child 1993; 69: 64-67)

Neonatal Unit,

Simpson Memorial

Maternity Pavilion,

Edinburgh

$S$ Cunningham

$S$ Deere

N McIntosh

Correspondence to:

Dr Steven Cunningham

Department of Child Life

and Health, University of

Edinburgh, 17 Hatton Place,

Edinburgh EH9 1UW.

Accepted 12 February 1993

We have noted cyclical variation in blood pressure and heart rate in neonates in intensive care and attempted to identify aetiological or predisposing factors.

The maintenance of normal blood pressure in neonates in intensive care is important to optimise the perfusion of vital organs. Blood pressure maintained within the 'normal' range
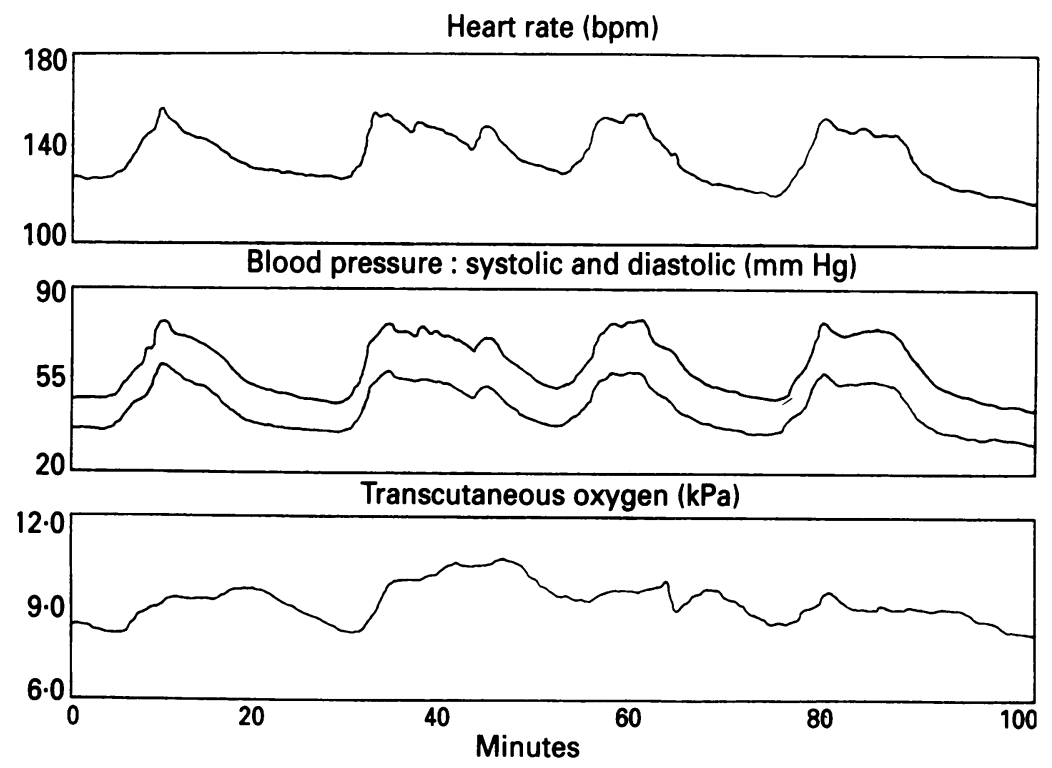

Figure 1 Cyclical variation of heart rate, blood pressure, and transcutaneous oxygen. reduces the risk of hypoxic ischaemic damage and may decrease the incidence of intraventricular haemorrhage. ${ }^{1}$

The normal adjustment of blood pressure is complex, involving neural, cardiovascular, and endocrine components. The opposing actions of the sympathetic and parasympathetic nervous systems are the dominant blood pressure controls. Sympathetic activity from spinal nerve centres increases heart rate, ventricular contractility, and peripheral vascular resistance. It is far more powerful in altering blood pressure than parasympathetic control mediated by carotid baroreceptors. ${ }^{2}$ In normal physiological states respiration may minimally alter blood pressure, but significant alterations of blood pressure can occur in certain pathological states, including cerebral asphyxia, hypovolaemia, and increases in cerebrospinal fluid pressure.

The blood pressure waves we describe may give some insight into cardiovascular control mechanisms in the neonate, and because of the prematurity of some of the infants, the fetal development of this system.

\section{Patients and methods}

All infants who required neonatal intensive care at the Simpson Memorial Maternity Pavilion since January 1991 were monitored using a computerised physiological cot monitoring system. ${ }^{3}$ Each infant in intensive care was monitored by a Hewlett Packard $78834 \mathrm{~A}$ multichannel monitor that was connected to a standard industry $316 \mathrm{SX}$ computer by means of an analogue to digit board. Data was sampled each second by the computer and up to 32 channels of physiological data could be accessed. The information was stored and could be shown as trend graphs over variable time periods from seven minutes to three days.

Cyclical oscillations ('waves') in blood pressure were first observed in the trend recorded data displayed on the computer of a severely asphyxiated neonate. The same oscillations were also present in the heart rate and transcutaneous oxygen. A sample of the data is shown in fig 1 . Four distinct waves can be seen in the heart rate, blood pressure, and transcutaneous oxygen. This episode occurred at 17 hours of age and continued for 23 hours.

Over the next five months, March to July 1991, we observed similar blood pressure waves in nine other neonates. To identify possible causes we considered the neonatal course of these 10 neonates (table 1). Daily intracranial ultrasound was performed including measurement of internal carotid artery 
Table 1 Patient details

\begin{tabular}{|c|c|c|c|c|c|c|c|c|}
\hline \multirow{2}{*}{$\begin{array}{l}\text { Gestation } \\
\text { (weeks) }\end{array}$} & \multirow{2}{*}{$\begin{array}{l}\text { Birth } \\
\text { weight } \\
\text { (g) }\end{array}$} & \multirow[b]{2}{*}{ Asphyxia } & \multicolumn{2}{|c|}{ Apgar score } & \multirow{2}{*}{$\begin{array}{l}\text { Grade of } \\
\text { intraventicular } \\
\text { haemorrhage }\end{array}$} & \multirow{2}{*}{$\begin{array}{l}\text { Resistance } \\
\text { indext }\end{array}$} & \multirow{2}{*}{$\begin{array}{l}\text { Neonatal } \\
\text { death }\end{array}$} & \multirow{2}{*}{$\begin{array}{l}\text { No of } \\
\text { episodes }\end{array}$} \\
\hline & & & $1 \min$ & $5 \mathrm{~min}$ & & & & \\
\hline 28 & 1129 & Postnatal & 6 & 9 & 3 & 0.55 & No & 1 \\
\hline 30 & 1755 & No & 7 & 5 & 0 & 0.69 & No & 1 \\
\hline 30 & 1770 & No & 6 & 7 & 0 & $0 \cdot 89$ & No & 1 \\
\hline 30 & 948 & Postnatal & 4 & 7 & 2 & 0.63 & No & 7 \\
\hline 31 & 1358 & Postnatal & 7 & 9 & 0 & - & No & 1 \\
\hline 35 & 1900 & Birth & 0 & 5 & 0 & 0.56 & No & 1 \\
\hline 38 & 2735 & Postnatal & 9 & 9 & 0 & 0.55 & No & 7 \\
\hline 41 & 3886 & Birth & 0 & 0 & 0 & 0.35 & Yes & 1 \\
\hline 41 & 2430 & Postnatal & 2 & 6 & 0 & & Yes & 1 \\
\hline 42 & 3125 & Birth & 2 & 4 & 4 & 0.30 & Yes & 4 \\
\hline
\end{tabular}

^Based on Papille et al grades 1 to $4 .^{18}$

†Internal carotid artery resistance index $\leqslant 0.55$ is considered abnormal. ${ }^{19}$

Table 2 Comparison of amplitude ( $\mathrm{mm} \mathrm{Hg}$ ) and wavelength (min) during the wave episodes

\begin{tabular}{lr}
\hline & Mean (SD) \\
\hline Blood pressure $(\mathrm{n}=25):$ & \\
$\quad$ Amplitude & $11 \cdot 6(5 \cdot 6)$ \\
$\quad$ Wavelength & $9 \cdot 0(5 \cdot 2)$ \\
Heart rate $(\mathrm{n}=23):$ & \\
$\quad$ Amplitude & $9 \cdot 4(6 \cdot 3)$ \\
Wavelength & $9 \cdot 2(5 \cdot 4)$ \\
Transcutaneous oxygen $(\mathrm{n}=17):$ & $0 \cdot 8(0 \cdot 7)$ \\
Amplitude & $7 \cdot 4(6 \cdot 1)$ \\
Wavelength & \\
\hline
\end{tabular}

resistance index. Eighteen other variables of neonatal intensive care were considered retrospectively from casenotes.

Electromechanical artefact might produce a regular variation in blood pressure. However, the blood pressure waves described have occurred over a five month period on a variety of computers, monitors, and infusion pumps and we can determine no artefact that could cause such regular variation, especially one that can be altered just by touching the baby.

Each episode of waves was analysed to obtain the mean systolic and mean diastolic blood pressure (values quoted in later text are a mean of these two values), heart rate, and transcutaneous oxygen over at least 15 cycles. The phase relation between each of the three indices was also noted.

Ethical permission for the use of computer monitoring was obtained from the Lothian Health Board medical ethics committee.

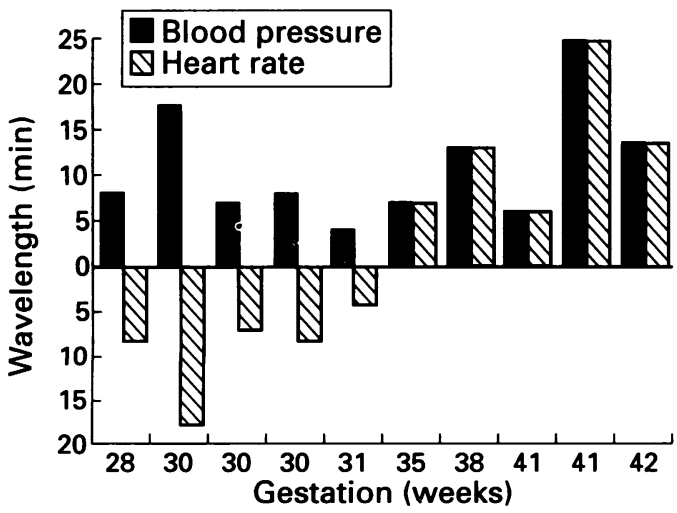

Figure 2 Maximum wavelength and phase relation of both blood pressure and heart rate in the 10 neonates.

Heart rate is plotted above the zero line when the heart rate and blood pressure are in phase (peak for peak and trough for trough). When the blood pressure peak coincides with a heart rate trough (that is the opposite phase) the heart rate is shown below the zero line.

\section{Results}

A total of 25 episodes were seen in 10 ventilated neonates over the five month period. Seven neonates had just one episode, one had four episodes, and two neonates had seven episodes. The episodes had a median duration of five hours with a range from one to 38 hours. These 25 episodes occurred at a median of 101 hours of age with a range from 17-315 hours. The 10 neonates (table 1 ) had a median gestational age of 33 weeks (range $28-42$ weeks) and birth weight median of $1835 \mathrm{~g}$ (range of 986-3886 g). Apgars scores were low, reflecting the presence of three severely birth asphyxiated neonates in the cohort. Five other neonates had postnatal asphyxia; this was defined by blood gases as an arterial oxygen tension of less than $5 \mathrm{kPa}$ in conjunction with a base deficit more severe than $10 \mathrm{mmol} / \mathrm{l}$ for more than 10 minutes.

The mean and SD of the amplitude and wavelength of the blood pressure, heart rate, and transcutaneous oxygen are shown in table 2. The largest mean amplitude and wavelength recorded in blood pressure waves were 25 $\mathrm{mm} \mathrm{Hg}$ and 25 minutes respectively. Heart rate and transcutaneous oxygen waves were not always associated with waves in blood pressure. Heart rate waves were associated in $92 \%$ of blood pressure waves and transcutaneous oxygen waves associated in $68 \%$ of blood pressure waves.

The wavelength, amplitude, and time of onset of the waves were unrelated to the gestation, birth weight, or Apgar scores. Benzylpenicillin, gentamicin, and morphine were commonly associated with the episodes. Hypocalcaemia (serum calcium concentration $<1.8 \mathrm{mmol} / \mathrm{l}$ ) was the only biochemical factor of those we analysed to be commonly associated (seven out of 10 neonates). Blood volume support was needed by five infants during their wave episodes; this increased to nine neonates when the period of 48 hours before and up to six hours after the wave episodes were included.

The phase relation of the blood pressure and heart rate waves is demonstrated in fig 2 , where the maximum blood pressure wavelength is plotted with the maximum heart rate wavelength for each of the 10 infants. Heart rate waves occurred only either directly in phase or directly in opposite phase to blood pressure waves. In fig 1, the blood pressure and heart rate waves are directly in phase, that is peaks correspond to peaks and troughs to troughs, whereas if they were in opposite phase blood pressure peaks would correspond to heart rate troughs and vice versa. In fig 2 heart rate wavelength is plotted above the midline if they occurred in phase with blood pressure waves or below the midline if in opposite phase. This figure demonstrates that infants less than 32 weeks' gestation have an inverse phase relation between heart rate and blood pressure waves, that is when the blood pressure increases the heart rate decreases and vice versa. Neonates greater than 34 weeks' gestation have a direct phase relation between heart rate and blood pressure waves, that 
is heart rate increases as blood pressure increases.

\section{Discussion}

Blood pressure is modulated by several major physiological controls, including the autonomic nervous system, the endocrine system, and the cardiovascular system. These combine to produce a blood pressure that varies little from its physiological norm, providing stability of perfusion essential for the function of major organs. Blood pressure deviates from this norm when this balance is pathologically interrupted such that other contributing controls cannot compensate.

The embryological development of human gross cardiovascular anatomy is well described, from the initial pumping of a single chambered heart at 23 days' gestation to the final transition from the fetal to newborn circulation at birth. The embryological development of the control of the cardiovascular system remains less well understood. ${ }^{4}$ Preterm neonates are now being cared for at little more than half their intended gestation. It is possibly unreasonable to assume that cardiovascular control is complete at these early gestations, when the fine control of all other major organs is not. Despite this we often assume adult blood pressure control mechanisms when managing a 4 month premature infant.

CYCLICAL NATURE OF BLOOD PRESSURE WAVES The synchronous relationship between the blood pressure and heart rate wavelengths (table 2), would indicate that these waves both share the same origin. Numerous postulates could account for this exact chronicity: the cardiovascular system is controlled by a number of neural and endocrine sites, many of which have an intrinsic rhythm that underlies their control. These rhythms may be exaggerated or have their frequency altered by hypoxia. ${ }^{5}$ A sinusoidal pattern of heart rate in the fetus has been demonstrated on cardiotocography ${ }^{6}$ in association with hypoxia and severe anaemia with a subsequent poor prognosis. A similar sinusoidal pattern in heart rate was reported postnatally in eight neonates suffering from perinatal or postnatal asphyxia. ${ }^{7}$ Only two of the eight survived, but with severe handicap. It is not stated whether blood pressure measurements were taken and no conclusions were made as to the origin of the heart rate waves.

Baroreceptors provide an important blood pressure control. Oscillations in blood pressure very similar to those we describe have been induced by sectioning the afferent nerve supply from arterial and cardiopulmonary baroreceptors in non-anaesthetised dogs. 8 The blood pressure waves produced in the dogs had a wavelength of approximately 20 minutes and an amplitude up to 80 $\mathrm{mm} \mathrm{Hg}$. Hypoxia of the same pathways in the human neonate might reduce their feedback function revealing an underlying intrinsic rhythm. ${ }^{9}$ Removal of $20 \%$ of the blood volume from a pig fetus induces 4 per minute waves in blood pressure and heart rate. ${ }^{10}$ Severe antepartum haemorrhage in the human neonate has also produced the cardiotocography cyclical heart rate rhythm described above. Blood volume support was needed in five of the neonates in this study during their wave episodes.

Endocrine feedback often shows cyclical variability - for example glucagon, insulin, and thyrotrophin release. ${ }^{11}$ Longitudinal data on neonatal endocrine function is scanty because of the difficulties (technical and ethical) of repetitive blood sampling in neonates. New microassays may enable a better appreciation of the temporal relationships of humoral control of blood pressure in neonates.

Cerebrospinal fluid (CSF) pressure waves are well described. ${ }^{12}$ Of the three types of pressure wave, ' $a$ ' and ' $b$ ' waves, with a wavelength of 5-20 minutes and 2-4 minutes respectively, are pathological and episodic, resulting from a temporary reduction in intracerebral compliance, whereas ' $c$ ' waves are physiological and are thought to occur secondary to the well described 6 per minute Traub Herring and Mayer blood pressure waves. Short term changes in intracranial pressure noted during CSF pressure ' $a$ ' waves are sometimes conveyed to the systemic blood pressure, ${ }^{12}$ others have been unable to confirm this. ${ }^{13}$ CSF ' $a$ ' waves have been described in asphyxiated neonates. ${ }^{14}$ These waves occurred only at intervals, in one case occurring on five of the 10 days of life and lasting for periods of up to seven hours with an amplitude of up to $60 \mathrm{~mm} \mathrm{Hg}$. The CSF pressure waves in these infants occurred both spontaneously and in response to external stimuli (for example suction). No reference is made to continuous blood pressure recordings in these neonates, but if the CSF pressure waves were conveyed to the systemic blood pressure, waves such as those we have described would be produced. Cerebral epileptic activity is often associated with variability of blood pressure and heart rate. We can find no reports of epilepsy in neonates leading to cyclical blood pressure waves. Sleep patterns might explain the periodic nature of the blood pressure waves. However, as neonates spend most of each 24 hours asleep, a large proportion of which is rapid eye movement sleep, it would seem unlikely that only single episodes of the blood pressure waves would be manifest if they are sleep induced.

From this study we have been unable to identify any factors that might have convincingly induced the waves or cause them to disappear. Changes in serum biochemistry and medication can clearly alter cardiovascular responsiveness and might therefore have induced temporary alteration in cardiovascular function producing a release of an intrinsic rhythm.

\section{PHASE RELATION OF BLOOD PRESSURE WAVES} The phase relation of blood pressure and heart rate waves undergoes a transition at 32-34 
weeks. Figure 2 demonstrates both the maximum wavelength of each of the 10 neonates and uses this to illustrate the phase relation that exists between blood pressure and heart rate. Mammalian models show a consistent asynchrony in the development of the autonomic control of the cardiovascular system; the sympathetic system is the principle control of the cardiovascular system by 0.5 term gestation and keeps control until near term. At this stage the parasympathetic nervous system, until now relatively dormant, markedly increases its contribution. The change in phase relation that we have demonstrated may herald a distinct period of change in the control mechanisms of blood pressure in the human neonate. Frequent bradycardias in preterm infants may be due to a parasympathetic system attempting to increase its share of control before it is physiologically mature. ${ }^{15}$

Immature infants lack the cardiac reserve of term neonates; myocardial contractility and sympathetic tone are already at near maximum. It is likely therefore that in the very immature cardiovascular system an increase in heart rate may reduce stroke volume and thus cardiac output. This might account for the change in phase relation of heart rate and blood pressure between the term and preterm infants. ${ }^{1617}$

\section{CONCLUSIONS}

Our main concern in gestations less than 32 weeks is that instability of blood pressure may predispose to intraventricular haemorrhage. The blood pressure waves may have an amplitude equivalent to $50 \%$ of resting blood pressure and so may represent a significant tension on the frail blood vessels of the germinal matrix. At later gestations the waves are frequently associated with birth asphyxia or a significant degree of postnatal asphyxia. Asphyxial loss of cerebral autoregulation and ongoing vasogenic and cytotoxic oedema may be compounded by damage from hypertensive blood pressure waves. A hypertensive encephalopathy could exacerbate the cerebral pathology.

1 Miall-Allen VM, De Vries LS, Whitelaw AGL. Mean arterial blood pressure and neonatal cerebral lesions. Arch Dis Child 1987; 62: 1068-9.

2 Gebber GL. Brainstem systems involved in cardiovascular regulation. In: Randall WC, ed. Neural regulation of the heart. New York: Oxford University Press, 1977: 346-68.

3 Cunningham S, Deere S, Elton RA, McIntosh N. Neonatal physiological trend monitoring by computer. Int $f$ Clin Monit Comput 1992; 9: 221-7.

4 Versmold HT. Control of blood pressure and the distribution of blood flow. Int $\mathcal{F}$ Technol Assess Health Care 1991; 7 (suppl 1): 79-84.

5 Kocsis B, Fedina L, Pasztor E. Two phase change of sympathetic rhythms in brain ischemia, Cushing reaction, and asphyxia. Am ₹ Physiol 1989; 256: R120-32.

6 Baskett TF, Koh KS. Sinusoidal fetal heart pattern. Obstet Gynecol 1974; 44: 379-82.

7 McC, Reid M, Jenkins J, McClure G. Sinusoidal heart rate rhythms in severe neonatal hypoxia. Arch Dis Child 1979; 54: 432-5.

8 Persson PB, Ehmke H, Köhler WW, Kirchheim HR. Identification of major slow blood pressure oscillations in conscious dogs. Am f Physiol 1990; 259: H1050-5.

9 Waldman S, Krauss AN,. Auld PAM. Baroreceptors in preterm infants: their relationship to maturity and disease. Dev Med Child Neurol 1979; 21: 714-22.

10 MacDonald AA. Blood pressure control and the developing pig fetus. University of Utrecht: Utrecht University Press, 1983. (Thesis.)

11 Lang DA, Matthes DR, Burnett M, Ward GM, Turner RC Pulsatile, synchronous basal insulin and glucagon secretion in man. Diabetes 1982; 31: 83-8.

12 Lundberg N. Continuous recording and control of ventricular fluid pressure in neurosurgical practice. Acta Psychiatric et Neurologica Scandinavica 1960; 36: suppl 149

13 Hayashi M, Kobayashi H, Handa Y, et al. Systemic blood pressure and respiration during plateau wave phenomena. No To Shinkei 1985; 37: 249-54.

14 Raju TNK, Vidyasagar D, Papazafiratou C. Cerebral perfusion pressure and abnormal intracranial pressure wave forms: their relation to outcome in birth asphyxia. Crit Care Med 1981; 9: 449-53.

15 Lagercrantz H, Edwards D, Henderson-Smart D, Hertzberg $\mathrm{T}$, Jeffery $\mathrm{H}$. Autonomic reflexes in preterm infants. Acta Paediatr Scand 1990; 79: 721-8.

16 Winberg P, Ergander U. Relationship between heart rate, left ventricular output, and stroke volume in preterm infants during fluctuations in heart rate. Pediatr Res 1992; 31: 117-20.

17 Agata Y, Hiraishi S, Oguchi K, et al. Changes in left ventricular output from fetal to early neonatal life. $\mathcal{f}$ Pediatr 1991; 119: 441-5.

18 Papille LA, Burnstein J, Burnstein R, Koffler H. Incidence and evolution of subependymal and intraventricular hemorrhage: study of infants with birthweight less than

9 Archer LNJ, Levene MI, Evans DH. Cerebral artery Doppler ultrasonography for prediction of outcome after perinatal asphyxia. Lancet 1986; ii: 1116-7. 